\title{
PERSPECTIVES
}

OPINION

\section{Cancer stem cell definitions and terminology: the devil is in the details}

Peter Valent, Dominique Bonnet, Ruggero De Maria, Tsvee Lapidot, Mhairi Copland, Junia V. Melo, Christine Chomienne, Fumihiko Ishikawa, Jan Jacob Schuringa, Giorgio Stassi, Brian Huntly, Harald Herrmann, Jean Soulier, Alexander Roesch, Gerrit Jan Schuurhuis, Stefan Wöhrer, Michel Arock, Johannes Zuber, Sabine Cerny-Reiterer, Hans E. Johnsen, Michael Andreeff and Connie Eaves

Abstract | The cancer stem cell (CSC) concept has important therapeutic implications, but its investigation has been hampered both by a lack of consistency in the terms used for these cells and by how they are defined. Evidence of their heterogeneous origins, frequencies and their genomic, as well as their phenotypic and functional, properties has added to the confusion and has fuelled new ideas and controversies. Participants in The Year 2011 Working Conference on CSCs met to review these issues and to propose a conceptual and practical framework for CSC terminology. More precise reporting of the parameters that are used to identify CSCs and to attribute responses to them is also recommended as key to accelerating an understanding of their biology and developing more effective methods for their eradication in patients.

Cancer stem cells (CSCs) have been the subject of intensive discussion and investigation for decades. Not surprisingly, what is meant by the term CSC has evolved over that period. Historically, it first invoked the concept that malignant cell populations are organized as unidirectional cellular hierarchies in which CSCs constitute biologically unique subsets of cells, which are distinguished from the bulk of the cells that they produce by their exclusive ability to perpetuate the growth of a malignant cell population indefinitely ${ }^{1-4}$. This concept is of considerable clinical importance because it emphasizes the crucial need to target CSCs to achieve cures. It also offers a possible explanation as to why many treatments seem to be effective initially but the patient later relapses. The implication is that CSCs are often innately less sensitive to treatment than are the bulk of the tumour cells that they generate. As a result, the tumour may initially appear to be eliminated, but it later reappears because the rarer and grossly invisible populations of
CSCs have survived. In addition to the inherent insensitivity of some CSCs to currently used anticancer drugs, continuing genomic and epigenomic changes are believed to confer new treatment-resistant properties that are then passed on to daughter generations of CSCs and their progeny ${ }^{5-9}$. Interactions of CSCs with their microenvironment (CSC niches) may also contribute to an ability to resist treatment ${ }^{9-13}$.

These clinically important issues have stimulated intense interest in experimental approaches for investigating CSCs. They point to a particular need for better methods to identify and distinguish individual human tumours in which a unidirectional hierarchy is evident at diagnosis and those in which such a hierarchy does not seem to be in place. This lack of hierarchy arises when all or most of the tumour cells have CSC properties, independently of their evolving genotype or phenotype, or when the mechanisms that regulate the CSC state have become unstable (reversible).
More recently, the term CSC has become associated with the operational detection of cells with CSC properties. In this case, the term refers to cells shown (retrospectively) to have been capable of generating and propagating a malignant cell population, usually identified and characterized in an experimental setting. Given the potential importance of information that could be derived from experimental systems and assays that are able to detect CSCs in cancer patients, there is now considerable interest in both the value of available methods and the properties and responses of the cells that they identify.

Current approaches use transplanted immunodeficient mice to characterize the ability of CSCs to regenerate malignant populations that resemble the parental cancer in vivo. This powerful strategy has been successfully applied to the detection of CSCs in many types of human cancers ${ }^{1-4,9,12}$. Sphere formation in non-adherent cultures has been used as a surrogate in vitro method for detecting CSCs from some primary human tumours $^{14-18}$. This in vitro approach has the advantage of being cheaper and faster, but also has the shortcoming of being reliant on a less physiological end point. Nevertheless, advances made using both approaches suggest their usefulness for developing more direct CSC detection methods that might be clinically meaningful. Examples include phenotypic and transcriptional marker profiles, assuming that these can be found to show sufficient stability, generality and specificity. However, given the extent of the genomic and phenotypic heterogeneity of malignant populations now known to characterize tumours of the same histological appearance and grade (including the CSCs that they contain), this goal is posing a considerable challenge.

The Year 2011 Working Conference on CSCs was organized to review and discuss these issues. The key points of discussion and the conclusions that were reached are summarized below.

\section{CSC definitions and terminology}

Stem cells in normal tissues are generally defined as undifferentiated cells that have the capacity to produce the specialized end cells of that tissue, as well as undifferentiated 
descendant stem cells with tissue-maintaining potential. This paradigm was first established in the haematopoietic system in mice and humans and has since been extended to many other tissues in both species ${ }^{3}$. Interestingly, it has also become apparent that even stringently defined normal tissue stem cells with extensive self-renewal properties can be intrinsically heterogeneous with regard to their differentiation and selfrenewal control in different sites or stages of development ${ }^{19-21}$. Thus, heterogeneity among CSCs in a single tumour type, or even within a single tumour, cannot be assumed to be indicative of an abnormal (tumour-specific) process or to be a consequence of a DNA mutation. Indeed, altered function of epigenetic regulators is rapidly emerging as a very prevalent feature of malignant populations ${ }^{6}$.

The idea that normal tissues are organized as fairly fixed developmental hierarchies derives from the assumption that differentiation involves the sequential activation of a series of molecular processes that coordinate discrete changes in a unidirectional and highly organized manner ${ }^{22}$ (FIG. 1). This model is based on the observed consistency of the process under homeostatic conditions. Because the changes may span multiple cell divisions, the process may allow separable subtypes of transit-amplifying cells to be distinguished ${ }^{23-25}$. Accordingly, normal tissue cells at sequential early stages of differentiation can now often be discriminated with great precision by immunological or molecular profiling - much greater than is achievable by morphology.

Phenotypic descriptions of normal tissue hierarchies have thus been powerful tools that enable direct analyses of changes that accompany, or that are even part of, the normal differentiation process. However, as yet, in no instance has a profile been identified that can reliably replace functional assays for normal stem cells. It should also be noted that many of the phenotypic markers found to be empirically useful for isolating particular subsets of cells in unperturbed normal tissue have proved to be functionally dispensable or differently regulated when they are activated in vivo or in vitro ${ }^{3,4}$. In addition, there is increasing evidence that normal tissue stem cells that are identified by their tissue-regenerative activity in different assays may fail to include cells with similar latent potentialities that are only revealed under different conditions. Examples of this situation have recently been documented for several epithelial tissues in the mouse, including the skin, mammary gland and intestine ${ }^{21}$. Memory $\mathrm{T}$ and $\mathrm{B}$ lymphocytes also represent anomalous differentiated cells that seem to have a very extensive innate self-renewal ability. Thus, exactly when a normal tissue cell loses the molecular machinery required to allow it to function like a stem cell is no longer as clearcut as the original concept of unidirectional differentiation suggested.

Tumours are known to arise from single cells in specific tissues through a series of genetic and/or epigenetic events that begin by interfering with mechanisms that normally control a stable physiological cell output in that tissue or that initiate a genomically unstable state ${ }^{5-8}$. This has given weight to the idea that cancer typically evolves from abnormal clones that, at least initially, preserve many features of the hierarchical structure of the normal tissue in which the malignancy has arisen (FIG. 1).
An important feature of normal tissue hierarchies is the tight linkage between observed differentiation-associated transcriptional, phenotypic and morphological changes and an accompanying loss of proliferative ability. Observations of early stage (pre-malignant) conditions suggest that these usually maintain many of the hallmarks of normal differentiation. At this stage, clonal amplification seems to reflect an increased self-renewal and proliferative activity and/or survival of primitive cells with continued differentiation and the production of large numbers of cells that are incapable of further division. The CSC concept implies that the production of cells that are incapable of further division is often retained even when more readily detected indicators of differentiation are blocked or lost.

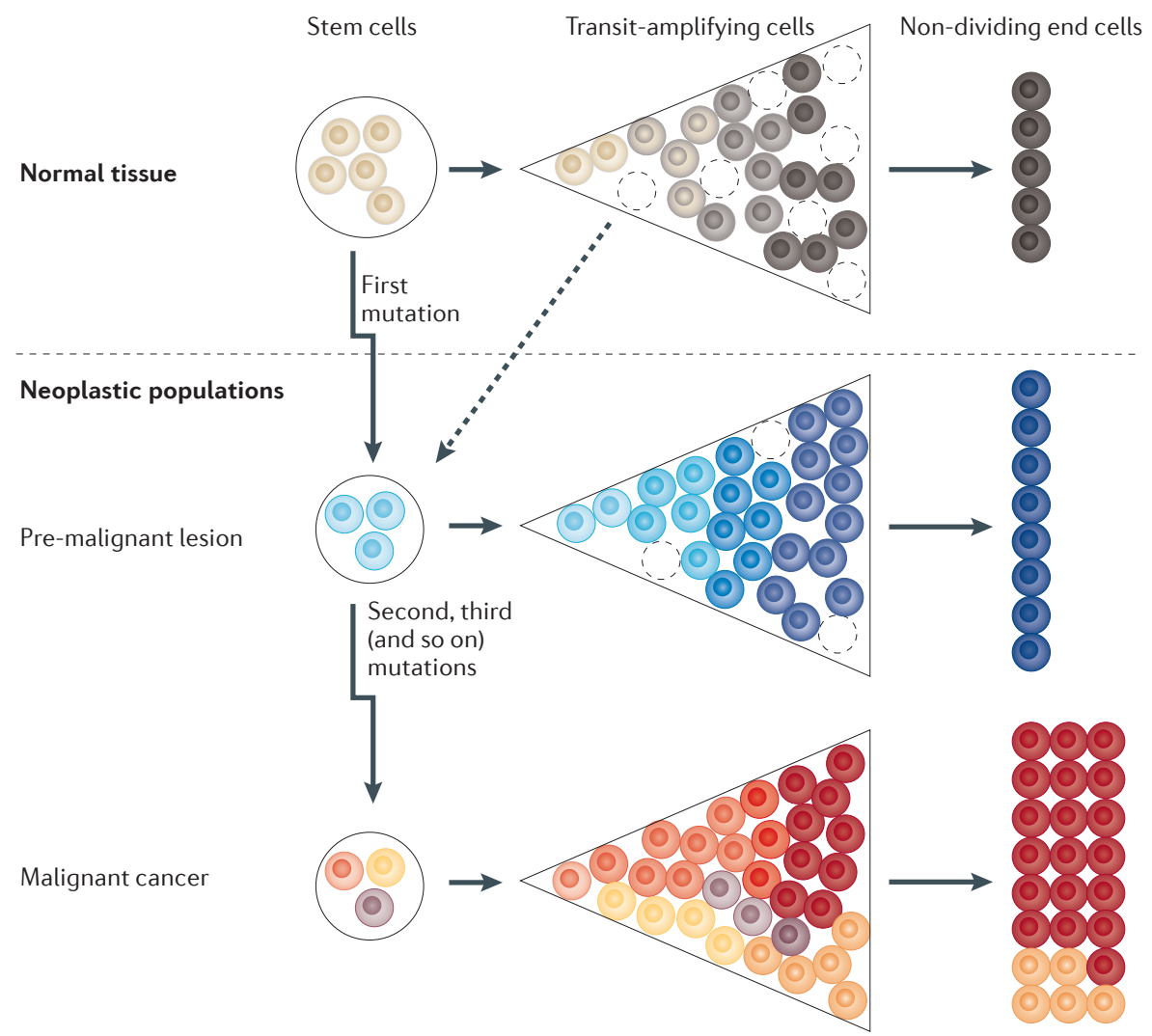

Figure 1 | Models of cancer stem cell evolution: perturbation of the normal differentiation hierarchy. A schematic comparison is shown of the highly coordinated and quantitatively regulated hierarchy of normal tissue populations in which cell turnover is a feature of tissue maintenance (top of figure), with stem cells giving rise to transit-amplifying cells that are differentiating (shown by dashed circles), to finally produce the mature cells of the tissue that are incapable of further division. During the evolution of malignant populations, the effects of alterations imposed by the sequential acquisition of mutations and/or epigenetic changes (indicated by the colour change) will first deregulate cell production and then lead to clonal expansion (middle of figure). Ultimately, a full-blown malignant clone may be produced. Such cells have typically acquired a complex profile of aberrations (indicated by multiple colours) and a loss of many features of normal differentiation characteristic of the transitamplifying compartment (bottom of figure). In this particular schema, neoplastic stem cells are shown as developing from normal stem cells, although multiple alternative scenarios in which neoplastic stem cells arise from pre-malignant cells with 'later' phenotypes or biology can be envisaged (dashed arrow). 
The term neoplasia is widely used to cover all scenarios in which a clone of cells appears to display perturbed growth properties. This contrasts with other abnormal conditions that represent reactive (polyclonal) responses to an external stimulus (although the development of a neoplastic clone may emerge from a prior polyclonal response). Neoplasias thus include pre-malignant and overtly malignant conditions. Pre-malignant neoplastic conditions are usually characterized by a greater (but usually unpredictable) clonal stability than malignant diseases. Premalignant conditions also have no immediate cancer-generating potential. As a result, their subsequent clinical course is also unpredictable. Nevertheless, they may, over time, progress to a full-blown malignant state with invasive properties. Examples include monoclonal gammopathy of unknown significance (MGUS), early stages of myeloproliferative neoplasms (MPNs), (pre-malignant) colonic adenoma, Barrett's oesophagus, pre-invasive dysplasia and in situ carcinoma. Conversely, most spontaneously arising human malignancies ${ }^{5-8}$ (although not all $^{26}$ ) are thought to represent subclonal derivatives that arise slowly and that evolve progressively from pre-malignant clones (FIG. 2).

Accordingly, we propose that neoplastic stem cells should be classified according to the same principles that have historically been established for neoplastic populations (TABLE 1). Neoplastic stem cells would thus include both pre-malignant neoplastic stem cells and malignant neoplastic stem cells, and only the malignant neoplastic stem cells would meet the definition of CSCs. Both types of neoplastic stem cells would thus refer exclusively to cells with the potential to propagate (maintain) a neoplastic population for a prolonged or unlimited period, and the term CSCs would be retained exclusively for cells that produce an overt invasive tumour or leukaemia.

These definitions make no inference about the normal cell in which the process of transformation was initiated, nor about the cell type in which a malignant state might first have been achieved. Indeed, they accommodate the possibility that disease progression can occur independently in different members of a pre-malignant clone ${ }^{7}$ (FIG. 2). They also imply that pre-malignant neoplastic stem cells have self-renewal capacity and produce daughter cells with limited proliferative potential.

An important feature of neoplastic stem cells is their extensive genomic (subclonal) heterogeneity. This is evident both in comparisons of different patients' tumours that

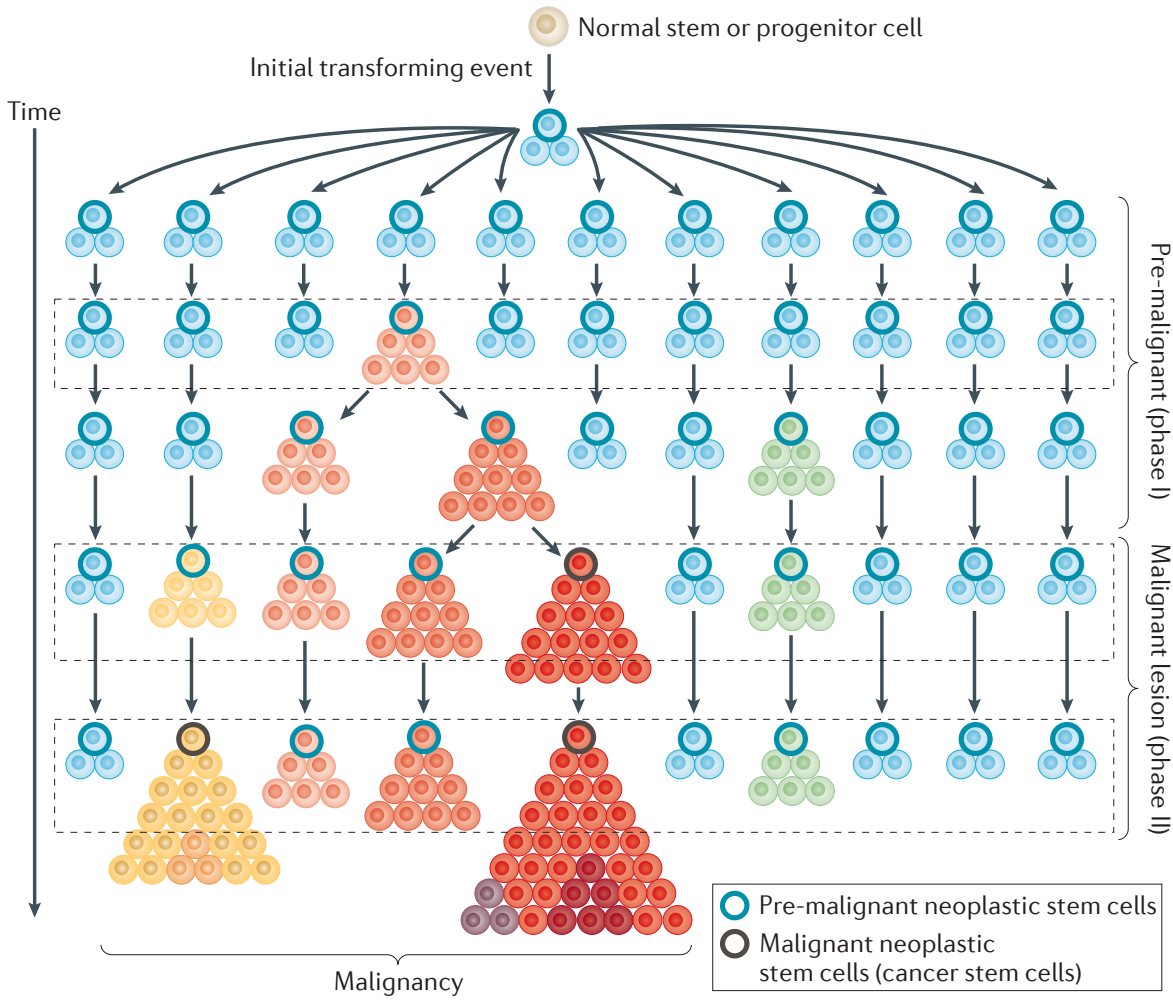

Figure 2 | Proposed model of cancer stem cell evolution. A schematic representation is shown of the continuously branching network of subclones envisaged to accompany the expansion of an initially mutated or epigenetically altered cell, thereby gaining a proliferative advantage over its normal counterparts that is sufficient to create a persistent neoplastic clone. This initial clone may produce distinct subclones that are controlled by the natural immune system and/or the environment of the surrounding tissue or organ (dashed rectangles) and hence these do not produce an overt malignancy. In this first phase of cancer evolution (phase I), neoplastic stem cells (indicated by blue outlines) are pre-malignant stem cells. However, if one or more subclones acquires a particular profile of hits (indicated by colour changes), an overt malignancy develops. At that time (phase II), the neoplastic stem cells become cancer-initiating cells and can thus be called cancer stem cells (CSCs) (indicated by black outlines). However, the other neoplastic subclones and their stem cells may remain and continue to generate additional pre-malignant and/or malignant subclones. The term pre-malignant (or pre-leukaemic) neoplastic stem cells is thus best confined to cells shown retrospectively (or experimentally) to be forerunners of malignant stem cells, whereas those that may never transform further are more accurately referred to simply as neoplastic (pre-malignant).

are classified as similar morphologically, and in a single tumour ${ }^{5-8,27}$. Such heterogeneity reflects the accrual and transmission of varying combinations of molecular lesions and oncogenic pathways that operate in continuously diversifying subclones (FIG. 2). These subclones have varying malignant potentials because of the molecular heterogeneity of their propagating stem cells and the different microenvironmental interactions that they may experience. A corollary to this view is that any persistent cancer subclone will contain a compartment of neoplastic stem cells with a unique genomic and epigenomic profile, recognizing that each subclone that develops may be a source of future new subclones with somewhat altered properties. As soon as one of the cells within such a subclone undergoes sufficient further change to acquire fully malignant potential, it becomes a CSC that, itself, may generate further altered CSCs (FIG. 2).

When the term CSC is used in an operational, rather than a conceptual, context, additional issues become important, as well as the nomenclature used. The terms cancer initiating, cancer propagating, cancer maintaining and repopulating have often been used to describe experimentally detected CSCs because these terms seem to offer greater specificity. However, they can also be a source of confusion. The term cancer initiating does not clearly distinguish between the original cells from which the malignancy first arose and the cells in a given suspension that are capable of producing malignant daughter cells in a given assay. However, the term cancer initiating 


\section{Table 1 | Terms to define cancer stem cells and required associated information}

Type of cells
Conceptual context
$\begin{aligned} & \text { Pre-malignant neoplastic } \\ & \text { stem cells }\end{aligned}$
$\begin{aligned} & \text { Cancer stem cells (malignant } \\ & \text { neoplastic stem cells) }\end{aligned}$

\section{Definition}

A subclass of neoplastic stem cells that can propagate neoplastic clones that may or may not develop into cancer stem cells over time, but that have no immediate cancer-initiating potential

A subclass of neoplastic stem cells that propagate malignant clones indefinitely and produce an overt cancer

Operational context

Neoplasia-initiating cells (in vivo)

Tumour or leukaemiainitiating cells

Neoplastic long-term culture-initiating cells

Neoplastic sphere-forming cells

Cells that regenerate detectable neoplastic ${ }^{*}$ populations in xenografted immunodeficient mice that are sustained ${ }^{\ddagger}$, usually measured by LDA

A subclass of neoplastic stem cells that regenerate detectable, sustained ${ }^{\ddagger}$ malignant* ${ }^{\star}$ populations in xenografted immunodeficient mice, usually measured by LDA

Cells that can initiate the sustained ${ }^{\S}$ production of neoplastic* populations when cultured in supportive conditions (for example, with stromal cells); can include pre-malignant and malignant neoplastic cells, usually measured by LDA

Cells that can initiate the production of non-adherent 'spheres' of neoplastic progeny in in vitro cultures, can include pre-malignant and malignant neoplastic cells, usually measured by counting spheres that can generate secondary spheres when re-plated (assumes spheres can be shown to be clones)

LDA, limiting dilution analysis. * Defined by obviously abnormal biological features exhibited by cells in the primary sample (for example, the formation of a palpable growth or tumour; the production of leukaemic

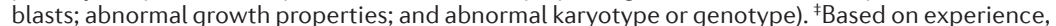
adapting this approach to normal stem cells, in this context sustained usually means $\geq 16$ weeks, and ideally demonstrable activity on serial transplantation into secondary mice. ${ }^{\S}$ Based on experience, adapting this approach to normal stem cells, in this context sustained usually means $\geq 6$ weeks with stromal cells \pm stimulatory growth factors.

does have the advantage of being a variant of similar terms that have already been established in the stem cell literature; for example, as in the terms, long-term culture-initiating cell (LTC-IC) $)^{28}$, neurosphere-initiating cell (NS-IC) ${ }^{29}$ or mammosphere-initiating cell $(\mathrm{M}-\mathrm{IC})^{30}$. But the term cancer-initiating cell should then be confined in its application to cells that are shown to initiate the formation of a progressively growing malignant population. The terms cancer propagating or cancer maintaining may be more accurate, at least in the context of xenotransplantation models; and the term long-term cancer propagating (or cancer maintaining) may help to further qualify the cells detected. The term repopulating has the advantage of implying an in vivo read out, but fails to highlight the cancer aspect of the repopulation and seems misleading, as it is a normal tissue of the host that is being repopulated with malignant cells. However, all of these terms highlight the need to designate the follow-up time adopted, or specify the use of serial transplantation experiments to meet the criterion of long term.

These considerations further highlight the importance of clearly specifying the protocol details that are associated with any term used to refer to an operationally identified CSC entity (TABLE 1). The discriminating power and hence the utility of the term CSC lies in the validity of the assumptions and inferences that are associated with its use. These, in turn, can only be examined and appreciated if the experimental details used to define a CSC as such are explicit.

\section{When a clonal hierarchy is in question}

The idea that malignant cell populations are hierarchically organized seems logical given the evidence for their stepwise evolution from cells in normal tissues. Experimental evidence for human CSCs is considered to be best established by demonstrating the ability of cells that are directly isolated from patients to produce malignant daughter populations in transplanted immunodeficient mice. This approach was pioneered with samples of cells from patients with various types of leukaemia (acute lymphoid leukaemia (ALL) ${ }^{31}$, acute myeloid leukaemia $(\mathrm{AML})^{32}$ and chronic myeloid leukaemia $\left.(\mathrm{CML})^{33}\right)$. Subsequently, the same approach has been successfully applied to solid tumours, including human cancers arising in the breast ${ }^{34}$, brain ${ }^{14}$, colon $^{16,35}$, ovary ${ }^{36}$, lung ${ }^{17}$, and head and neck ${ }^{37}$. One criterion used to establish that these CSCs are biologically different from the bulk of the cells in the tumour has been the demonstration that they have a distinct phenotype that allows their prospective and selective enrichment. However, in most cases, the phenotype of cells meeting the very stringent functional definition of a CSC (tumour-initiating activity in an immunodeficient mouse at the single-cell level) has not proved to be singular or even stable.

For example, $\mathrm{CD} 34^{+} \mathrm{CD} 38^{+} \mathrm{AML}$ cells, as well as $\mathrm{CD} 34^{+} \mathrm{CD} 38^{-}$AML cells, both frequently show CSC activity when transplanted into non-obese diabetic, severe combined immunodeficient (NOD/SCID) mice lacking a functional IL2RG chain (NSG or NOG) mice, although previously, this property seemed to be mostly restricted to the $\mathrm{CD} 34^{+} \mathrm{CD} 38^{-}$subset when less immunodeficient NOD/SCID mice were used as recipients $\mathrm{s}^{38,39}$. Nevertheless, a hierarchy of cells can still be found to exist within these leukaemias ${ }^{40}$. In addition, perturbations of differentiation that affect normal lineage restriction processes (for example, as found in biphenotypic leukaemias), or the production of abnormal phenotypes, are well documented consequences of leukaemogenesis ${ }^{41}$. Similar examples of coexisting or unstable CSC phenotypes have also been reported in human $\mathrm{ALL}^{42}$ and several types of human solid tumours ${ }^{36,43-45}$. These findings highlight the fact that malignant cells have perturbed gene expression programmes that might affect the normal stability or the developmental control of the expression of certain cell surface markers, particularly when the cells are put in vitro. Thus phenotype data alone have not proved sufficient to infer the existence of a developmental hierarchy or the presence of a CSC.

In some malignancies, even functional xenotransplantation evidence of a persisting hierarchical structure in a malignant population is not apparent. Advanced human malignant melanoma is one such example ${ }^{46}$. For such malignancies, the utility of experimental assays for CSCs per se, will have weaker clinical relevance. Nevertheless, the use of xenografts to evaluate their responses to novel treatments in vivo remains an attractive possibility. Such advanced tumours do not negate the validity of the CSC concept, as tumorigenesis is known to be a multistep process in which more aggressive subclones typically supplant a preceding clone in which a hierarchy did exist.

It has recently been suggested that the very molecular mechanisms that confer CSC properties on cells within malignant clones; that is, the CSC state, may also be 
unstable ${ }^{47-50}$. This is an intriguing idea particularly in light of the growing number of major regulators of the epigenome that are commonly altered in malignant populations ${ }^{51}$. In addition, we now know that only a handful of oncogenes can turn almost any mammalian cell into an embryonic stem cell (ESC)-like cell, which often retains tumorigenic potential even after participating in normal embryogenesis ${ }^{52}$. However, whether reprogramming has an important role in the generation and evolution of human tumours is unknown and will also be very challenging to investigate, as functional assays attribute a CSC state exclusively to cells that display a sustained tumour-generating ability (in vivo).

\section{CSC assays: strengths and caveats}

To date, immunodeficient mice have proved the most sensitive recipients for supporting the growth of many sources of human CSCs and have made it possible to develop in vivo assays to detect and quantify these cells. However, the ability or failure of a cell to produce a detectable malignant population in a transplanted mouse might not accurately reflect its behaviour in a cancer patient. The limitations of currently available xenotransplantation models and possibilities for overcoming these limitations are shown in TABLE 2. Continued genetic modifications of candidate host mice will probably enable at least some of the species-specific needs to be addressed in the future. These include the possibility of creating transgenic mice that will express species-specific human cytokines, or the use of humanized mice (mice transplanted with human haematopoietic or local tissue components) to create more human-type microenvironments - as has proved useful for certain types of normal human cells $\mathrm{s}^{53-57}$. Orthotopic injections of CSCs into various target organs may also prove useful, and mimicry of natural tumour immunosurveillance mechanisms may be achievable using injections of specific immune effector cells (TABLE 2).

Attempts have also been made to quantify CSCs on the basis of their demonstrated or presumed self-renewal activity in vitro. For leukaemias, the systems used to select for the most primitive cells have typically applied conditions that were developed for their normal counterparts. These involve co-culturing the cells on a stromal cell feeder layer ${ }^{28}$ (or on feeder cells engineered to produce stimulatory growth factors ${ }^{58,59}$ ), and the use of an end point that measures continued progenitor output (for example, the LTC-IC assay). For solid tumours, the most popular end point has been sphere formation in non-adherent culture containers $^{14-18}$. However, such assays also have obvious caveats. One caveat is that factors important for the in vivo growth and self-renewal of some or all CSCs may not be provided by the in vitro conditions used. As a result, the cells being tested may show no or selected growth, and/or may be anomalously and rapidly induced to differentiate (enter a nondividing end state) without prior expansion. Additionally, it is unlikely that either of these in vitro systems fully replicates the three-dimensional structure and environment of an in vivo tumour. Furthermore, the growth of malignant cells in vitro is usually limited to several weeks or months, during which time many cells typically differentiate or die, or transform into a clinically irrelevant population. Therefore, results obtained from such in vitro assays may be less useful for deriving clinically meaningful predictions.

Many human tumour cell lines, or primary tumour cells adapted for efficient maintenance in culture, have also been used as model sources of CSCs assayed by phenotype or growth assays in vitro or in transplanted mice. However, the extent to

\section{Table 2 | Limitations of xenotransplant assays of human cancer stem cells}

\begin{tabular}{|c|c|}
\hline Limitations & Possible solutions \\
\hline Short lifetime of mice & Second-generation recipients or other animals \\
\hline Altered homing of CSCs & $\begin{array}{l}\text { Orthotopic injection, humanized vasculature, } \\
\text { humanized organs, humanized mice and } \\
\text { growth-supporting synthetic scaffolds embedded in } \\
\text { local tissue sites }\end{array}$ \\
\hline $\begin{array}{l}\text { Lack of cytokines that can stimulate } \\
\text { growth of CSCs }\end{array}$ & $\begin{array}{l}\text { Injection of cytokines, cytokine-transgenic mice, } \\
\text { humanized organs and humanized mice }\end{array}$ \\
\hline $\begin{array}{l}\text { Lack of tumour-specific } \\
\text { microenvironment }\end{array}$ & $\begin{array}{l}\text { Local injection of human microenvironmental cells, } \\
\text { humanized mice and supportive scaffolds }\end{array}$ \\
\hline $\begin{array}{l}\text { Lack of intact immune cells; lack of } \\
\text { natural immunosurveillance }\end{array}$ & $\begin{array}{l}\text { Prior transplantation of } \mathrm{CD} 34^{+} \text {haematopoietic cells, } \\
\text { humanized lymphoid organs, humanized mice and } \\
\text { co-transplanation of immune cells (for example, NK cells) }\end{array}$ \\
\hline
\end{tabular}

which the behaviour of such cells reflects the original genotype or tumour of origin is difficult to ascertain. Accordingly, considerable caution needs to be exercised in the types of questions in vitro passaged cells are used to address. Conversely, meta-analyses of cancer cell line responses to known anticancer drugs illustrate how these can be used to derive potential associations between some drug responses and the tissue of origin and genotype of the tumour cell line $e^{60}$.

In experimental settings, details that define the methods and end points used are clearly important. In particular, seemingly innocuous parts of protocols, such as the duration and method of tissue dissociation, may influence the subsets (subclones) of CSCs that are detected. Similarly, when malignant cell lines are studied, the line should be specifically identified (for example, HCT116-propagating cells), and not assigned a more generic term (such as colon CSCs).

Experimental mouse models have also been invaluable contributors to progress in understanding the nature of CSCs. In this case, the role of specific genes in the spontaneous generation of tumours can be examined using transgenic technology, as well as transplantation assays that can use syngeneic recipients of any age. More recently, lineage tracing has been exploited to add further strong evidence of CSCs in glioblastomas, intestinal adenomas and epidermal tumours developing de novo in mice and, in the case of the glioblastoma, their anticipated drug resistance was also demonstrated ${ }^{61-63}$. These findings highlight how mouse models of tumorigenesis may facilitate the future development of methods for interrogating CSCs in patient samples for clinical purposes.

CSC niche: definition and open questions The concept of stem cell niches arises from observations that stem cells have discrete locations in tissues in vivo. Most CSCs are derived from cells in normal tissues in which local cues have an important regulatory role and have not yet acquired completely autonomous growth ability. In addition, recent evidence indicates that CSCs respond to antitumour agents differently in vitro and in vivo, reinforcing the concept that the niche in which a CSC is located may be an important determinant of how it responds to a given treatment. Several different cell types have been implicated in forming such niche environments, including fibroblasts, endosteal cells in the bone marrow, perivascular and vascular cells, and tissue 
macrophages ${ }^{3,11}$. However, little is known about the particular contributions of any of these cells, or how their effects on CSCs might be mediated at a molecular level. Whether, and to what extent, CSCs may contribute to important features of their microenvironment through autocrine or paracrine mechanisms, or even by creating clonal niche components, is also unclear.

Molecules anticipated to be involved in CSC-niche interactions include certain adhesion receptors, cytokine receptors, membrane-bound and soluble cytokine ligands, and various chemotactic factors. A noteworthy example is CXCR4, a receptor of stromal cell-derived factor 1 (SDF1; also known as CXCL12). CXCR4 is expressed on many cancer cells, and SDF1 is a nichederived chemoattractant for CXCR4 $4^{+}$cells, thereby enhancing their entry into the bone marrow ${ }^{64}$. These observations have led to the development of clinically effective drugs that inhibit CXCR4-SDF1 interactions ${ }^{65,66}$ and that may be used to mobilize CSCs and possibly to sensitize them to therapy ${ }^{67}$. Conversely, CSCs often exhibit an acquired defect in their interaction with their specific microenvironments, leading to an abnormal or accelerated dissemination and metastasis formation ${ }^{64}$. CSC-niche interactions may also contribute to the acquisition of more aggressive properties by cells in solid tumours through the promotion of a response referred to as an epithelial-to-mesenchymal transition (EMT).

\section{Identifying therapeutic targets in CSCs}

Principal modes of action of currently used anticancer agents on CSCs and the resulting (theoretical) outcomes are illustrated in FIG. 3 and Supplementary information S1 (figure). Mechanisms that contribute to the responses exhibited by CSCs to known drugs are detailed in Supplementary information

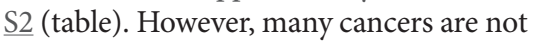
effectively eliminated by these strategies. Thus, a major goal in CSC research is to devise new strategies that will kill CSCs. To date, most efforts have focused either on searches for targets expressed in CSCs, but not in normal stem cells $\left(\mathrm{CD} 34^{+}\right.$cells in human leukaemias or $\mathrm{CD} 133^{+}$or $\mathrm{CD} 44^{+}$ cells isolated from solid tumours), or on screens for selective drugs or RNA interference (RNAi) molecules that selectively kill CSCs over normal stem cells. Candidate therapeutic targets identified include various receptors and their oncogenic derivatives, adhesion molecules, antibody-accessible surface components, signalling intermediates, survival pathway elements, chromatin modifiers and metabolic targets ${ }^{68-90}$. Gene expression profiling, next-generation sequencing and increasingly sophisticated screening methods are anticipated to extend these lists in the near future.

However, most of these studies are at a necessarily early stage given all of the caveats in current CSC research. These include the unreliability of phenotype end points as rapid indicators of CSC responses, the low purity of CSC-enriched populations currently obtainable directly from patients (typically less than a few percent) and the unreliability of expression biomarkers to predict functional consequences. A good example of this unreliability is provided by the inability of CML stem cells to be efficiently killed by drugs that target the BCR-ABL kinase, even though CML stem cells express BCR-ABL ${ }^{91,92}$. Finally, even if some CSCs are responsive, the target may not be expressed or may already be mutated in other CSCs that might not even be present at a detectable level ${ }^{91}$. This would allow the malignant cell population to escape a particular targeted therapy and produce a relapse (FIG. 3; Supplementary information S1 (figure)). Interactions between CSCs and their local microenvironment (niche) may also contribute to a lack of treatment response in vivo even when sensitivity is demonstrated in vitro. This has prompted interest in developing CSC-mobilizing strategies, to render them potentially more susceptible even to conventional treatments ${ }^{67}$.

\section{Evaluation of CSC eradication}

Although long-term disease-free survival after drug discontinuation might be considered to be a stringent (operational) indicator of clinical cure, solid criteria for complete disease eradication, and thus definitive cure, in cancer patients are difficult to define. In addition, the clinical need for complete disease eradication and the definition of cure have different meanings for individual patients. For example, aggressive approaches to eliminate all malignant cells may be counterindicated for patients with an otherwise (tumour-independent) limited life expectancy. Similarly, for patients who have diseases such as hairy cell leukaemia, the residual malignancy, including its stem cells, can remain indolent (pre-malignant) for years after the levels of tumour cells are initially reduced and, if they later increase, the cells are typically responsive to the same therapy as the original cells.

The use of xenotransplanted cells (for example, in NSG mice) is currently accepted as the best model for evaluating new drug candidates. However, the optimal experimental design is not clear. The simplest first approach is to expose the test cells to the drug of interest ex vivo and to subsequently assay its effect by comparing the rate (or frequency) of tumour growth in NSG mice that are transplanted with control versus treated cells. However, the outcome may not reflect the response that would be seen following an in vivo treatment after the tumour cells have been transplanted either before or after a regenerated malignant population has become detectable, depending on the magnitude of effect sought or expected. Complete and permanent disappearance of the malignant population after drug discontinuation would be a desirable outcome, but this is difficult to require as an experimental end point because of the cost and lack of knowledge as to whether a more rapid indicator might be sufficient.

Importantly, owing to the relatively short lifespan of mice, even apparent cures in this model may not be sufficient to be clinically relevant. Secondary or tertiary transplants can be used to test for surviving CSCs, but again the predictive relevance of the results is unknown. Furthermore, most xenotransplantation methods have restricted quantitative power, being generally limited to the detection of less than 100-fold reductions in CSC numbers, but lasting benefits in patients may require much larger reductions. Thus, even the most dramatic effects obtained in any transplant cancer model need to be interpreted with caution. This issue will probably become increasingly important in the face of emerging data on the vast genomic and epigenomic heterogeneity of many tumours ${ }^{6-8}$ indicative of subclones that would be well below the current limit of CSC detection. Conversely, in some instances, what may seem to be small (less than tenfold) reductions in tumour growth or CSC numbers in a transplant model, may prove of significant value clinically. Together, these issues highlight the emerging importance of correlative studies that might guide the development of predictive end points for particular tumour responses to specific agents.

In cancer patients, the possibility of detecting minimal residual disease (MRD) by sensitive molecular markers (for example, quantitative PCR) or other measures ${ }^{93}$ is an important aspect of disease management. However, depending on the type of neoplasm, persistent MRD may not necessarily argue against long-term disease-free survival. In AML, it has been reported that 
a
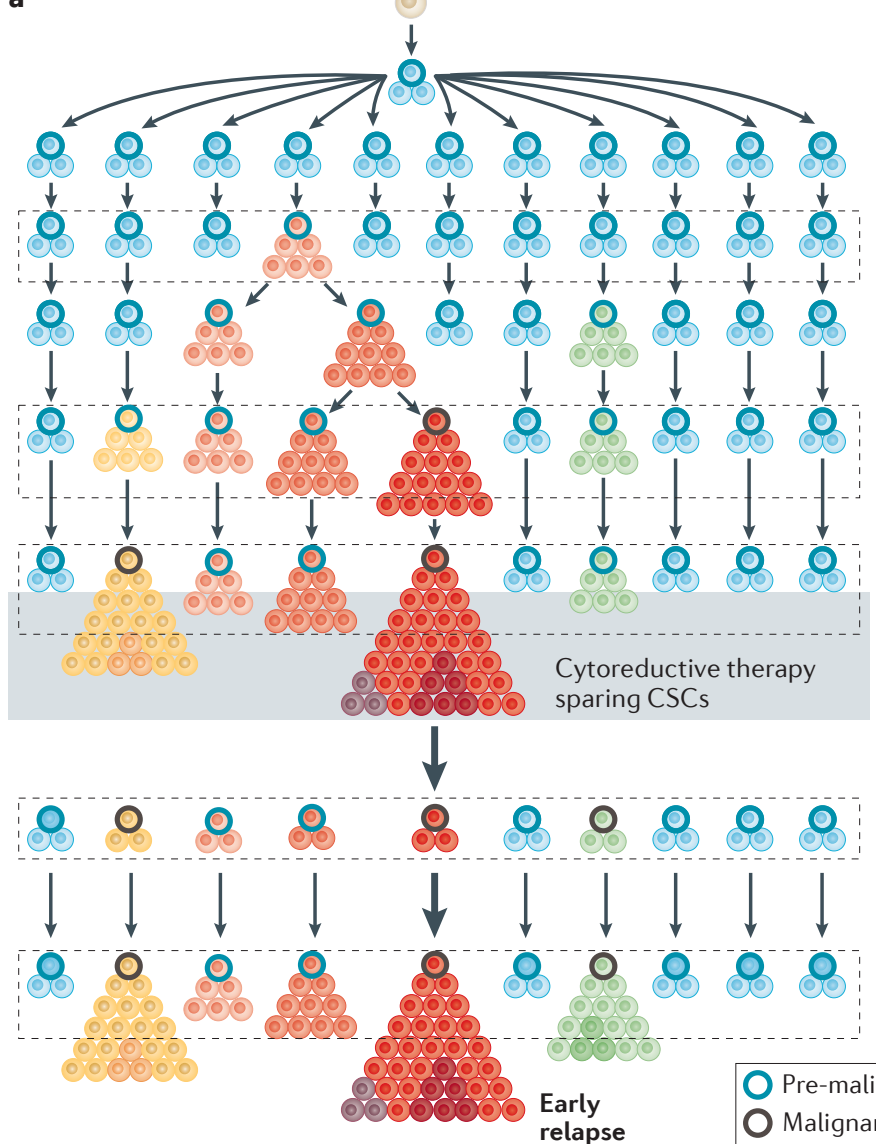

88 b

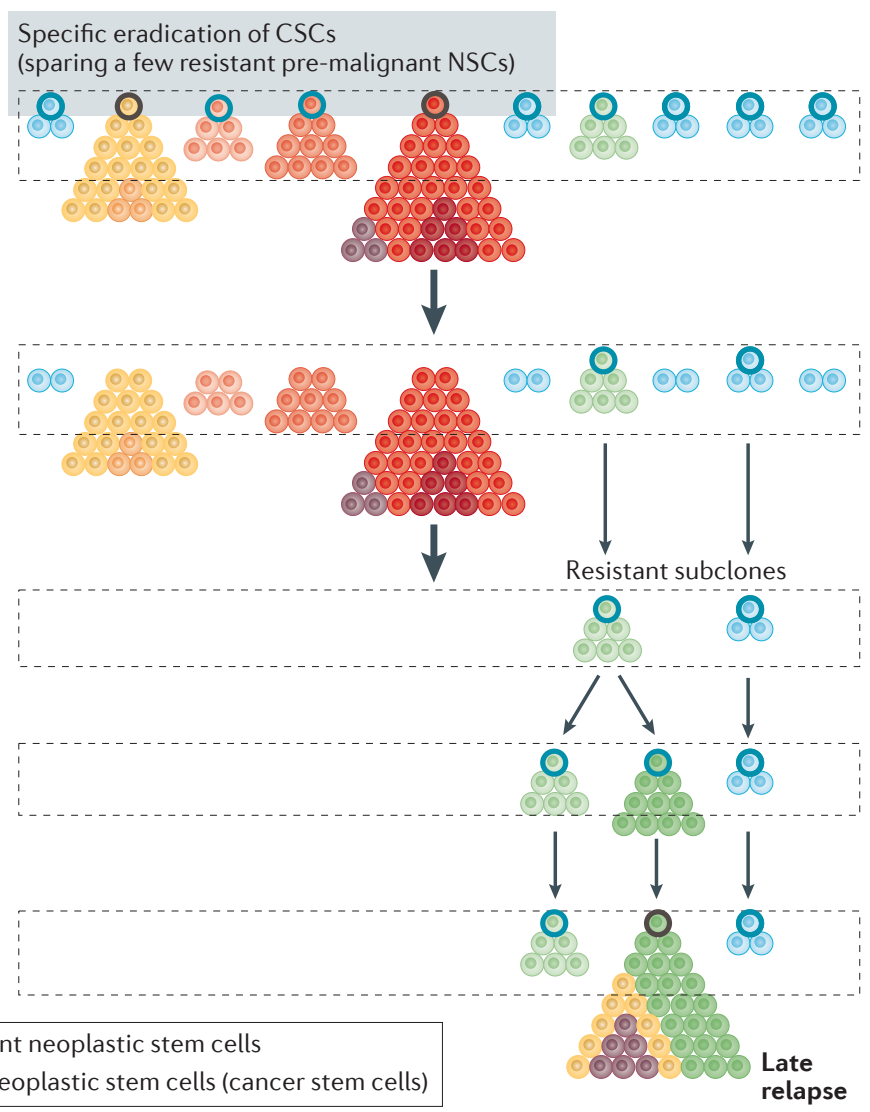

(sparing a few resistant pre-malignant NSCs)

relapse

Pre-malignant neoplastic stem cells
Malignant neoplastic stem cells (cancer stem cells)

Figure 3 | Effects of therapies. When most cancer stem cells (CSCs) survive therapy, an early relapse follows (part a). However, even after having successfully eradicated a dominant subclone (or even many other relevant subclones), the disease may relapse from a pre-malignant neoplastic stem cell (NSC) (part b). In this case late relapses may occur, with variable and unpredictable latency periods, depending on the time required for the accumulation of further hits in these subclone-specific (stem) cells to enable them to become fully malignant; that is, to transform into CSCs. Accordingly, relapses may occur months to decades later, and the relapsing cancer may display very different features and response to treatment. certain features of CSC-enriched populations correlate with clinical responses to conventional treatments ${ }^{94-97}$. Conversely, in chronic phase CML, the persistence of a detectable (that is, substantial) population of primitive (presumably pre-malignant) CML stem cells is not predictive of relapse ${ }^{98}$.

Routine assays for MRD used clinically may, however, be unable to distinguish a new (often indolent or pre-malignant) subclone from the original dominant one. Interestingly, non-malignant clones bearing the genetic hallmarks of malignant clones have been reported to be commonly present at detectable levels in normal individuals ${ }^{99-102}$. Whole-genome sequencing now offers an approach to discriminate different subclones and their clonal relationships and mode of evolution with unprecedented power, particularly if this methodology can be made usefully applicable to blood or other body fluids ${ }^{103}$. Thus, as sequencing costs decrease, it seems likely that such approaches will aid the use of MRD assessments to predict the likelihood of relapse.

\section{Summary}

The CSC hypothesis provides a model that accommodates the multiple causes of heterogeneity that characterize human malignancies as they evolve. Such heterogeneity exists not only between the same types of cancer arising in different individuals, but also within the malignant cell population that forms in an individual patient. This is due to the fact that the formation of a cancer involves the genesis and continuous diversification of subclones that become abnormally and non-homogeneously distributed, as they acquire an increasingly unstable control of many properties, including their ability to continue to proliferate. At an early stage in this process, a hierarchy that recapitulates features of the differentiation process of the tissue of origin is often still apparent.
The associated experimental observation that only a small, phenotypically distinguishable subset of the malignant cells is capable of generating a malignant population when transplanted into immunodeficient mice is the experimental basis of inferring the existence of a CSC population. The CSC concept is of key importance clinically because it predicts that cures require strategies that will eliminate CSC populations, as these cells sit at the top of any residual hierarchies within the malignant population. However, additional elimination of pre-malignant, as well as malignant, stem cells, is likely to be necessary in many patients to achieve a sustained disease-free outcome or even definitive cure.

Although experiments with many primary tumours suggest that they fit a CSC model, increasing complexity and diversity in the composition, location and behaviour of members of individual malignant populations has led to confusion and controversy about the utility and validity of the CSC 
concept. Many of these issues can be attributed to the multistep origin, and the now well-documented heterogeneity of CSCs that reflects the multitude of driver and passenger epigenetic and genetic perturbations that these cells accumulate during their evolution. As a result, phenotypic plasticity superimposed on a multiplicity of pre-malignant and malignant subclones are features now recognized as common in neoplastic populations by the time they become symptomatic. In some advanced neoplasms, these changes may result in the majority of the cells having overt or unstable CSC properties. At the opposite extreme are early stage cancer lesions, where the pre-malignant neoplastic stem cells may not already possess, and may not necessarily ever acquire, full CSC activity.

None of these observations negates the central value of the CSC concept. Rather, they highlight its importance as an element that is likely to be crucial in understanding the complex and patient-specific evolutionary process by which human malignancies develop their genetic and epigenetic diversity. Accordingly, the CSC concept is likely to have increasing importance as new tools for diagnosing cancers at earlier stages become available. In this respect, increased precision in the details of the methods used and described in identifying CSCs will be central to progress in improving patient management.

Peter Valent, Harald Herrmann and Sabine Cerny-Reiterer are at The Department of Internal Medicine I, Division of Hematology \& Hemostaseology, Medical University of Vienna \& Ludwig-Boltzmann Cluster Oncology, Waehringer Guertel 18-20, A-1090 Vienna, Austria.

Dominique Bonnet is at The Haematopoietic Stem Cell Laboratory, Cancer Research UK, London Research Institute, Lincoln's Inn Fields Laboratories, PO BOX 123, 44 Lincoln's Inn Fields, London WC2A 3LY, UK.

Ruggero De Maria is at The Regina Elena National Cancer Institute, Via Elio Chianesi 53, Rome 00144, Italy.

Tsvee Lapidot is at The Weizmann Institute of Science, Department of Immunology, Rehovot 76100, Israel.

Mhairi Copland is at The Paul O'Gorman Leukaemia Research Centre, College of Medical Veterinary and Life Sciences, University of Glasgow, Gartnavel General Hospital, 1053 Great Western Road, Glasgow G12 OZD, UK.

Junia V. Melo is at The Department of Haematology Centre for Cancer Biology, IMVS, Frome Road, Adelaide SA 5000, Australia and Imperial College London, Ducane Road, London W12 ONN, UK.

Christine Chomienne is at The Service de Biologie Cellulaire, UMR-S-940, Institut Universitaire d'Hématologie, Hôpital Saint Louis, 1 Avenue Claude Vellefaux 75010, Paris, France.
Fumihiko Ishikawa is at The Research Unit for Human Disease Models, RIKEN RCAI, Research Center for Allergy and Immunology, 1-7-22 Suehiro-cho Tsurumi-ku, Yokohama 230-0045, Japan.

Jan Jacob Schuringa is at The Department of Experimental Hematology, University Medical Center Groningen, University of Groningen, Hanzeplein 1, 9713 GZ Groningen, The Netherlands.

Giorgio Stassi is at The Laboratory of Cellular and Molecular Pathophysiology, Department of Surgical and Oncological Sciences, University of Palermo,

Via Liborio Giuffre', 5, 90127 Palermo, Italy.

Brian Huntly is at The Department of Haematology, Cambridge Institute for Medical Research, and Stem Cell Institute, University of Cambridge, Hills Road, Cambridge CB2 OXY, UK.

Jean Soulier is at The Institut National de la Santé et de la Recherche Médicale U944 and Haematology Laboratory, Hôpital Saint Louis and Université Paris Diderot, 1 Avenue Claude Vellefaux 75010 Paris, France.

Alexander Roesch is at The Saarland University Hospital, Department of Dermatology, Kirrbergstrasse, Building 18, D-66421 Homburg, Germany.

Gerrit Jan Schuurhuis is at The Department of Hematology, VU University Medical Center, De Boelelaan 1117, 1081 HV Amsterdam, The Netherlands.

Stefan Wöhrer is at The Medical University of Vienna, Department of Internal Medicine I, Bone Marrow and Stem Cell Transplantation Unit, Waehringerguertel 18-20, 1090 Vienna, Austria.

Michel Arock is at The LBPA CNRS UMR8113, Ecole Normale Supérieure de Cachan, 61 Ave du Président Wilson, 94235 Cachan CEDEX, France.

Johannes Zuber is at The Research Institute of Molecular Pathology, Vienna Biocenter, Dr. Bohr-Gasse 7, 1030 Vienna, Austria.

Hans E. Johnsen is at The Department of Haematology, Aalborg Hospital Science and Innovation Center, AHSIC Aarhus University Hospital, Sdr Skovvej 15, DK-9000 Aalborg, Denmark.

Michael Andreeff is at The Department of Leukemia and Department of Stem Cell Transplantation and Cellular Therapy, The University of Texas M. D. Anderson Cancer Center, 1515 Holcombe Boulevard, Houston TX 77030, USA.

Connie Eaves is at The Terry Fox Laboratory, British Columbia Cancer Agency, 601 West 10th Avenue, Vancouver BC V5Z 1L3, Canada. Correspondence to P.V. and C.E. e-mails: peter.valent@meduniwien.ac.at; ceaves@bccrc.ca doi: $10.1038 /$ nrc3368 Published online 11 October 2012

1. Reya, T., Morrison, S. J., Clarke, M. F. \& Weissman, I. L. Stem cells, cancer, and cancer stem cells. Nature 414, 105-111 (2001).

2. Clarke, M. F. et al. Cancer stem cells-perspectives on current status and future directions: AACR workshop on cancer stem cells. Cancer Res. 66, 9339-9344 (2006).

3. Nguyen, L. V., Vanner, R., Dirks, P. \& Eaves, C. J. Cancer stem cells: an evolving concept. Nature Rev. Cancer 12, 133-143 (2012).

4. Schulenburg, A. et al. Neoplastic stem cells: current concepts and clinical perspectives. Crit. Rev. Oncol. Hematol. 76, 2512-2520 (2010).

5. Nowell, P. C. The clonal evolution of tumor cell populations. Science 194, 23-81 (1976).
6. Baylin, S. B. \& Jones, P. A. A decade of exploring the cancer epigenome - biological and translational implications. Nature Rev. Cancer 11, 726-734 (2011).

7. Greaves, M. \& Maley, C. C. Clonal evolution in cancer. Nature 481, 306-313 (2012).

8. Stratton, M. R. Exploring the genomes of cancer cells: progress and promise. Science 331, 1553-1558 (2011).

9. Magee, J. A., Piskounova, E. \& Morrison, S. J. Cancer stem cells: impact, heterogeneity, and uncertainty. Cancer Cell 21, 283-296 (2012).

10. Singh, A. \& Settleman, J. EMT, cancer stem cells and drug resistance: an emerging axis of evil in the war on cancer. Oncogene 29, 4741-4751 (2010).

11. Konopleva, M. Y. \& Jordan, C. T. Leukemia stem cells and microenvironment: biology and therapeutic targeting. J. Clin. Oncol. 29, 591-599 (2011).

12. Visvader, J. E. \& Lindeman, G. J. Cancer stem cells: current status and evolving complexities. Cell Stem Cell 10, 717-728 (2012).

13. Gillies, R. J., Verduzco, D. \& Gatenby, R. A Evolutionary dynamics of carcinogenesis and why targeted therapy does not work. Nature Rev. Cancer 12, 487-493 (2012)

14. Singh, S. K. et al. Identification of human brain tumour initiating cells. Nature 432, 396-401 (2004).

15. Ponti, D. et al. Isolation and in vitro propagation of tumorigenic breast cancer cells with stem/progenitor cell properties. Cancer Res. 65, 5506-5511 (2005).

16. Ricci-Vitiani, L. et al. Identification and expansion of human colon-cancer-initiating cells. Nature $\mathbf{4 4 5}$ 111-115 (2007).

17. Eramo, A. et al. Identification and expansion of the tumorigenic lung cancer stem cell population. Cell Death Differ. 15, 504-514 (2008).

18. Han, M. E. et al. Cancer spheres from gastric cancer patients provide an ideal model system for cancer stem cell research. Cell. Mol. Life Sci. 68, 3589-3605 (2011).

19. Copley, M. R. Beer, P. A. \& Eaves, C. J. Hematopoietic stem cell heterogeneity takes center stage. Cell Stem Cell 10, 690-697 (2012).

20. Bixby, S., Kruger, G. M., Mosher, J. T., Joseph, N. M. $\&$ Morrison, S. J. Cell-intrinsic differences between stem cells from different regions of the peripheral nervous system regulate the generation of neural diversity. Neuron 35, 643-656 (2002).

21. Van Keymeulen, A. \& Blanpain, C. Tracing epithelial stem cells during development, homeostasis, and repair. J. Cell Biol. 197, 575-584 (2012).

22. Graf, T. \& Enver, T. Forcing cells to change lineages. Nature 462, 587-594 (2009).

23. Doulatov, S., Notta, F., Laurenti, E. \& Dick, J. E. Hematopoiesis: a human perspective. Cell Stem Cell 10, 120-136 (2012)

24. Smalley, M. J. et al. Isolation of mouse mammary epithelial subpopulations: a comparison of leading methods. J. Mammary Gland Biol. Neoplasia 17, 91-97 (2012).

25. Wagers, A. J. \& Conboy, I. M. Cellular and molecular signatures of muscle regeneration: current concepts and controversies in adult myogenesis. Cell 122, 659-667 (2005)

26. Stephens, P. J. et al. Massive genomic rearrangement acquired in a single catastrophic event during cancer development. Cell 144, 27-40 (2011).

27. Gerlinger, M. et al. Intratumor heterogeneity and branched evolution revealed by multiregion sequencing. N. Engl. J. Med. 366, 883-892 (2012).

28. Sutherland, H. J., Lansdorp, P. M., Henkelman, D. H. Eaves, A. C. \& Eaves, C. J. Functional characterization of individual human hematopoietic stem cells cultured at limiting dilution on supportive marrow stromal layers. Proc. Natl Acad. Sci. USA 87, 3584-3358 (1990).

29. Uchida, N. et al. Direct isolation of human central nervous system stem cells. Proc. Natl Acad. Sci. USA 97, 14270-14275 (2000).

30. Dontu, G. et al. In vitro propagation and transcriptional profiling of human mammary/stem progenitor cells. Genes Dev. 17, 1253-1270 (2003).

31. Kamel-Reid, S. et al. A model of human acute lymphoblastic leukemia in immune-deficient SCID mice. Science 246, 1597-1600 (1989).

32. Lapidot, T. et al. A cell initiating human acute myeloid leukaemia after transplantation into SCID mice. Nature 367, 645-648 (1994).

33. Sirard, C. et al. Normal and leukemic SCIDrepopulating cells (SRC) coexist in the bone marrow and peripheral blood from CML patients in chronic phase, whereas leukemic SRC are detected in blast crisis. Blood 87, 1539-1548 (1996). 
34. Al-Haji, M., Wicha, M. S., Benito-Hernandez, A., Morrison, S. J. \& Clarke, M. F. Prospective identification of tumorigenic breast cancer cells. Proc Natl Acad. Sci. USA 100, 3983-3988 (2003).

35. O'Brien, C. A., Pollett, A., Gallinger, S. \& Dick, J. E. A human colon cancer cell capable of initiating tumour growth in immunodeficient mice. Nature $\mathbf{4 4 5}$, 106-110 (2007)

36. Stewart, J. M. et al. Phenotypic heterogeneity and instability of human ovarian tumor-initiating cells. Proc. Natl Acad. Sci. USA 108, 6468-6473 (2011).

37. Prince, M. E. et al. Identification of a subpopulation of cells with cancer stem cell properties in head and neck squamous cell carcinoma. Proc. Natl Acad. Sci. USA 104, 973-978 (2007).

38. Taussig, D. C. et al. Anti-CD38 antibody-mediated clearance of human repopulating cells masks the heterogeneity of leukemia-initiating cells. Blood 112 , 568-575 (2008)

39. Taussig, D. C. et al. Leukemia-initiating cells from some acute myeloid leukemia patients with mutated nucleophosmin reside in the CD34 fraction. Blood 115, 1976-1984 (2010)

40. Goardon, N. et al. Coexistence of LMPP-like and GMPlike leukemia stem cells in acute myeloid leukemia. Cancer Cell 19, 138-152 (2011).

41. Weinberg, O. K. \& Arber, D. A. Mixed-phenotype acute leukemia: historical overview and a new definition. Leukemia 24, 1844-1851 (2010).

42. Kong, Y. et al. $\mathrm{CD} 344^{+} \mathrm{CD} 38{ }^{+} \mathrm{CD} 19+$ as well as CD34 ${ }^{+}$CD38 ${ }^{\circ D 19}$ + cells are leukemia-initiating cells with self-renewal capacity in human B-precursor ALL. Leukemia 22, 1207-1213 (2008).

43. Dirks, P. B. Brain tumor stem cells: the cancer stem cell hypothesis writ large. Mol. Oncol. 4, 420-430 (2010).

44. Shmelkov, S. V. et al. CD133 expression is not restricted to stem cells, and both $\mathrm{CD}_{133}{ }^{+}$and $\mathrm{CD} 13$ metastatic colon cancer cells initiate tumours. J. Clin. Invest. 118, 2111-2120 (2008).

45. Quintana, E. et al. Phenotypic heterogeneity among tumourigenic melanoma cells from patients that is reversible and not hierarchically organized. Cancer Cell 18, 510-523 (2010)

46. Quintana, E. et al. Efficient tumour formation by single human melanoma cells. Nature 456, 593-598 (2008).

47. Gupta, P. B., Chaffer, C. L. \& Weinberg, R. A. Cancer stem cells: mirage or reality? Nature Med. 15 1010-1012 (2009)

48. Gupta, P. B. et al. Stochastic state transitions give rise to phenotypic equilibrium in populations of cancer cells. Cell 146, 633-644 (2011)

49. Roesch, A. et al. A temporarily distinct subpopulation of slow-cycling melanoma cells is required for continuous tumour growth. Cell 141, 583-594 (2010).

50. Chaffer, C. L. et al. Normal and neoplastic nonstem cells can spontaneously convert to a stem-like state Proc. Natl Acad. Sci. USA 108, 7950-7955 (2011).

51. Baylin, S. B. \& Jones, P. A. A decade of exploring the cancer epigenome - biological and translational implications. Nature Rev. Cancer 11, 726-734 (2011).

52. Okita, K. \& Yamanaka, S. Induced pluripotent stem cells: opportunities and challenges. Philosoph. Trans. R. Soc. B. Biol. Sci. 366, 2198-2207 (2011)

53. Akkina, R. et al. Humanized Rag $1^{-/-} \gamma \mathrm{C}^{-/-}$mice support multilineage hematopoiesis and are susceptible to HIV-1 infection via systemic and vaginal routes. PLOS ONE 6, e20169 (2011).

54. Strowig, T. et al. Transgenic expression of human signal regulatory protein $\alpha$ in $\operatorname{Rag} 2^{-1-} \gamma \mathrm{C}^{-/-}$mice improves engraftment of human hematopoietic cells in humanized mice. Proc. Natl Acad. Sci. USA 108, 13218-13223 (2011).

55. Wunderlich, M. et al. AML xenograft efficiency is significantly improved in NOD/SCID-IL2RG mice constitutively expressing human SCF, GM-CSF and IL-3. Leukemia 24, 1785-1788 (2010).

56. Takagi, S. et al. Membrane-bound human SCF/KL promotes in vivo human hematopoietic engraftment and myeloid differentiation. Blood 119, 2768-2777 (2012).

57. Lan, P., Tonomura, N., Shimizu, A., Wang, S. \& Yang, Y. G. Reconstitution of a functional human immune system in immunodeficient mice through combined human fetal thymus/liver and CD34 ${ }^{+}$cell transplantation. Blood 108, 487-492 (2006).

58. Petzer, A. L. et al. Characterization of primitive subpopulations of normal and leukemic cells present in the blood of patients with newly diagnosed as well as established chronic myeloid leukemia. Blood $\mathbf{8 8}$ 2162-2171 (1996).
59. Blair, A., Hogge, D. E., Ailles, L. E., Lansdorp, P. M. \& Sutherland, H. J. Lack of expression of Thy-1 (CD90) on acute myeloid leukemia cells with long-term proliferative ability in vitro and in vivo. Blood $\mathbf{8 9}$, 3104-3112 (1997).

60. Garnett, M. J. et al. Systematic identification of genomic markers of drug sensitivity in cancer cells. Nature 483, 570-575 (2012).

61. Chen, J. et al. A restricted cell population propagates glioblastoma growth after chemotherapy. Nature $\mathbf{4 8 8}$ 522-526 (2012)

62. Schepers, A. G. et al. Lineage tracing reveals Lgr5 stem cell activity in mouse intestinal adenomas. Science 337, 730-735 (2012).

63. Driessens, G., Beck, B., Caauwe, A., Simons, B. D. \& Blanpain, $C$. Defining the mode of tumour growth by clonal analysis. Nature 488, 527-530 (2012).

64. Domanska, U. M. et al. A review on CXCR4/CXCL1 2 axis in oncology: no place to hide. Eur. J. Cancer 8 Jun 2012 [epub ahead of print].

65. Damon, L. E. \& Damon, L. E. Mobilization of hematopoietic stem cells into the peripheral blood Exp. Rev. Hematol. 2, 717-733 (2009).

66. Kessans, M. R., Gatesman, M. L. \& Kockler, D. R. Plerixafor: a peripheral blood stem cell mobilizer. Pharmacotherapy 30, 485-492 (2010).

67. Burger, J. A. \& Peled, A. CXCR4 antagonists: targeting the microenvironment in leukemia and other cancers. Leukemia 23, 43-52 (2009).

68. Jin, L., Hope, K. J., Zhai, Q., Smadja-Joffe, F. \& Dick, J. E. Targeting of CD44 eradicates human acute myeloid leukemic stem cells. Nature Med. 12 , 1167-1174 (2006).

69. Florian, S. et al. Detection of molecular targets on the surface of CD34 ${ }^{+} / \mathrm{CD} 38$ stem cells in various myeloid malignancies. Leuk. Lymphoma 47, 207-222 (2006).

70. Hosen, N. et al. CD96 is a leukemic stem cell-specific marker in human acute myeloid leukemia. Proc. Natl Acad. Sci. USA 104, 11008-11013 (2007).

71. Van Rhenen, A. et al. The novel AML stem cell associated antigen CLL-1 aids in discrimination between normal and leukemic stem cells. Blood 110 2659-2666 (2007)

72. Jin, L. et al. Monoclonal antibody-mediated targeting of CD123, IL-3 receptor a chain, eliminates human acute myeloid leukemic stem cells. Cell Stem Cell 5 31-42 (2009)

73. Järås, M. et al. Isolation and killing of candidate chronic myeloid leukemia stem cells by antibody targeting of IL-1 receptor accessory protein. Proc. Natl Acad. Sci. USA 107, 16280-16285 (2010)

74. Kemper, K., Grandela, C. \& Medema, J. P. Molecular identification and targeting of colorectal cancer stem cells. Oncotarget 1, 387-395 (2010).

75. Lorico, A. \& Rappa, G. Phenotypic heterogeneity of breast cancer stem cells. J. Oncol. 2011, 135039 (2011).

76. Korkaya, H. \& Wicha, M. S. Selective targeting of cancer stem cells: a new concept in cancer therapeutics. BioDrugs 21, 299-310 (2007).

77. Valent, P. Emerging stem cell concepts for imatinibresistant chronic myeloid leukaemia: implications for the biology, management, and therapy of the disease. Br. J. Haematol. 142, 361-378 (2008).

78. Tu, L. C., Foltz, G., Lin, E., Hood, L. \& Tian, Q. Targeting stem cells-clinical implications for cancer therapy. Curr. Stem Cell Res. Ther. 4, 147-153 (2009).

79. Gupta, P. B. et al. Identification of selective inhibitors of cancer stem cells by high-throughput screening. Cell 138, 645-659 (2009).

80. Curtin, J. C. \& Lorenzi, M. V. Drug discovery approaches to target Wnt signaling in cancer stem cells. Oncotarget 1, 563-577 (2010).

81. Pannuti, A. et al. Targeting Notch to target cancer stem cells. Clin. Cancer Res. 16, 3141-3152 (2010)

82. Martelli, A. M. et al. Targeting the phosphatidylinositol 3-kinase/Akt/mammalian target of rapamycin signaling network in cancer stem cells. Curr. Med. Chem. 18 2715-2726 (2011)

83. Allan, E. K., Holyoake, T. L., Craig, A. R. \& Jørgensen, H. G. Omacetaxine may have a role in chronic myeloid leukaemia eradication through downregulation of $\mathrm{Mcl}-1$ and induction of apoptosis in stem/progenitor cells. Leukemia 25, 985-994 (2011).

84. Takebe, N., Harris, P. J., Warren, R. Q. \& Ivy, S. P. Targeting cancer stem cells by inhibiting Wnt, Notch, and Hedgehog pathways. Nature Rev. Clin. Oncol. 8 97-106 (2011).

85. de Sousa, E. M., Vermeulen, L., Richel, D. \& Medema, J. P. Targeting Wnt signaling in colon cancer stem cells. Clin. Cancer Res. 17, 647-653 (2011).
86. Wei, L et al. Hsp27 participates in the maintenance of breast cancer stem cells through regulation of epithelial-mesenchymal transition and nuclear factor-kB. Breast Cancer Res. 13, R101 (2011)

87. Zuber, J. et al. RNAi screen identifies Brd4 as a therapeutic target in acute myeloid leukemia. Nature 478, 524-528 (2011).

88. Dawson, M. A. et al. Inhibition of BET recruitment to chromatin as an effective treatment for MLL-fusion leukemia. Nature 478, 529-533 (2011).

89. Skrtic, M. et al. Inhibition of mitochondrial translation as a therapeutic strategy for human acute myeloid leukemia. Cancer Cell 20, 674-688 (2011)

90. Sachlos, E. et al. Identification of drugs including a dopamine receptor antagonist that selectively target cancer stem cells. Cell 149, 1284-1297 (2012).

91. Valent, P. Targeting of leukemia-initiating cells to develop curative drug therapies: straightforward but nontrivial concept. Curr. Cancer Drug Targets 11, 56-71 (2011).

92. Barnes, D. J. \& Melo, J. V. Primitive, quiescent and difficult to kill: the role of non-proliferating stem cells in chronic myeloid leukemia. Cell Cycle 5, 2862-2866 (2006).

93. Irish, J. M., Kotecha, N. \& Nolan, G. P. Mapping normal and cancer cell signalling networks: towards single-cell proteomics. Nature Rev. Cancer 6 146-155 (2006)

94. Ho, M. M., Hogge, D. E. \& Ling, V. MDR 1 and BCRP1 expression in leukemic progenitors correlates with chemotherapy response in acute myeloid Leukemia. Exp. Hematol. 36, 433-442 (2008).

95. Rosen, D. B. et al. Distinct patterns of DNA damage response and apoptosis correlate with Jak/Stat and PI3 kinase response profiles in human myelogenous Leukemia. PLOS ONE 5, e 12405 (2010).

96. de Jonge, H. J. et al. Gene expression profiling in the leukemic stem cell-enriched CD34+ fraction identifies target genes that predict prognosis in normal karyotype AML. Leukemia 25, 1825-1833 (2011).

97. Eppert, K. et al. Stem cell gene expression programs influence clinical outcome in human leukemia. Nature Med. 17, 1086-1093 (2011).

98. Melo, J. V. \& Ross, D. M. Minimal residual disease and discontinuation of therapy in chronic myeloid leukemia: can we aim at a cure? Hematol. Am. Soc Hematol Educ. Program 2011, 136-142 (2011).

99. Liu, Y., Hernandez, A. M., Shibata, D. \& Cortopassi, G. A. BCL2 translocation frequency rises with age in humans. Proc. Natl Acad. Sci. USA 91, 8910-8914 (1994)

100. Limpens, J. et al. Lymphoma-associated translocation $\mathrm{t}(14 ; 18)$ in blood B cells of normal individuals. Blood 85, 2528-2536 (1995)

101. Biernaux, C., Loos, M., Sels, A., Huez, G. $\delta$ Stryckmans, P. Detection of major bcr-abl gene expression at a very low level in blood cells of some healthy individuals. Blood 86, 3118-3122 (1995).

102. Cazzaniga, G. et al. Developmental origins and impact of BCR-ABL1 fusion and IKZF1 deletions in monozygotic twins with $\mathrm{Ph}^{+}$acute lymphoblastic leukemia. Blood 118, 5559-5564 (2011).

103. Leary, R. J. et al. Development of personalized tumor biomarkers using massively parallel sequencing. Sci. Transl. Med. 2, 20ra14 (2010).

\section{Acknowledgements}

The Year 2011 Working Conference on Cancer Stem Cells was supported by a Cancer Stem Cell Grant of the Medica University of Vienna, Austria. The authors would like to thank S. Sonnleitner and K. Krassel for their helpful technical sup port. H.E.J. was supported by the EU 6th FP to MSCNET (LSHC-CT-2006-037602). M.C. is supported by a Fellowship from the Scottish Funding Council (SCD/04) and J.V.M. by the NIHR Biomedical Research Centre Funding Scheme, UK.

Competing interests statement

The authors declare no competing financial interests.

\section{FURTHER INFORMATION}

Peter Valent's homepage: http://www.meduniwien.ac.at/ haematology/index 2 .php?section=ma valent Connie Eaves' homepage:

http://www.terryfoxlab.ca/people/Ceaves/connie.aspx

SUPPLEMENTARY INFORMATION

See online article: $\mathbf{S 1}$ (figure) I $\underline{\text { 2 (table) }}$

ALL LINKS ARE ACTIVE IN THE ONLINE PDF 